\title{
EQ-5D Utilities in Chronic Spontaneous/Idiopathic Urticaria
}

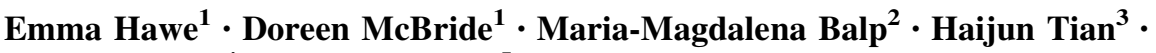 \\ Anna Halliday ${ }^{4}$. Donald E. Stull ${ }^{5}$ (i)
}

Published online: 20 January 2016

(c) The Author(s) 2016. This article is published with open access at Springerlink.com

\begin{abstract}
Objectives To obtain utility estimates suitable for use in economic models for chronic spontaneous (idiopathic) urticaria (CSU).

Methods Patient-level data from three randomized clinical trials-ASTERIA I, ASTERIA II and GLACIALwere analysed. Health states were derived from the Urticaria Activity Score over 7 days (UAS7); higher scores denote greater activity. The health state score ranges were urticaria free: 0 ; well-controlled urticaria: $1-6$; mild urticaria: 7-15; moderate urticaria: $16-27$; and severe urti-
\end{abstract}

Electronic supplementary material The online version of this article (doi:10.1007/s40273-015-0375-7) contains supplementary material, which is available to authorized users.

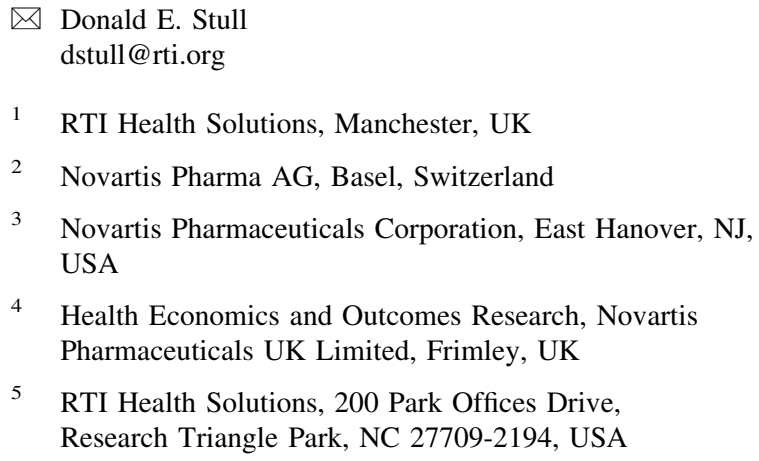

caria: 28-42. The mean EQ-5D utilities were calculated for each health state. A mixed model was used to predict the EQ-5D according to UAS7 health states in a pooled data set containing all treatment arms and time points from the three trials. Pooled trial data were validated through visual comparisons and interaction terms. Fixed and random effects for trials and patients were included, along with the following covariates: UAS7 health state at baseline (moderate or severe); presence of angioedema at baseline and during follow-up; duration of CSU; number of previous CSU medications; visit; current treatment; and patient age and sex.

Results There was a consistent improvement in EQ-5D utilities as urticaria activity decreased. The mean utilities ranged from 0.710 (severe urticaria) to 0.780 (moderate urticaria), 0.829 (mild urticaria), 0.862 (well-controlled urticaria) and 0.894 (urticaria free). Sensitivity and subgroup analyses confirmed the robustness of the results. Conclusion The results suggest that EQ-5D utility scores increase with decreasing urticaria activity. EQ-5D utility scores enable the health-related quality of life of CSU patients to be compared with that of patients with other diseases. 


\section{Key Points for Decision Makers}

Although an increasing amount of knowledge is available about the health-related quality of life of patients with chronic spontaneous (idiopathic) urticaria, no health state utilities have been established for this condition. Utilities are key data for economic models used in making reimbursement decisions in many countries.

This paper presents utilities for different levels of disease activity of chronic spontaneous urticaria. This information will be valuable for future costeffectiveness models. Utilities measured by generic measures, such as the EQ-5D, allow for healthrelated quality-of-life comparisons between different disease areas.

Evaluation of these utilities provides robust results for health technology assessment bodies to evaluate the cost effectiveness of treatments for chronic spontaneous urticaria and quantification of health gains by calculation of quality-adjusted life-years.

\section{Introduction}

Chronic spontaneous (also known as idiopathic) urticaria (CSU) is defined by the current European Academy of Allergy and Clinical Immunology/Global Allergy and Asthma European Network/European Dermatology Forum/ World Allergy Organization guidelines as the occurrence, for 6 weeks or longer, of itchy hives (wheals), angioedema, or both, due to known or unknown causes [1].

At any one time, up to $1 \%$ of the population have chronic urticaria [2], and CSU represents approximately two thirds of these cases [3]. Published data quantifying the burden of the condition are limited [4] but indicate that chronic urticaria can have a substantial impact on patients' health-related quality of life (HRQoL), their ability to perform daily tasks and their mental health $[1,5,6]$.

The activity of CSU is assessed by evaluating the signs (hives) and symptoms (itch) of the disease with a daily diary of the Urticaria Activity Score (UAS). Daily average UAS scores over 7 days are summed to a weekly UAS7 score. Changes in UAS7 scores provide a good measure of treatment efficacy, and improvements in UAS7 scores are reflected in significant improvements in HRQoL [7].

The use of the generic, preference-based EQ-5D instrument for measuring health utilities in economic evaluations is well understood. Within this study, the threelevel version of the EQ-5D was used [8], which generates
243 health states valued by the general population using the time trade-off method [9]; a five-level version has since been developed [10] but was not available when the data for these analyses were collected.

To our knowledge, there is no published information on the relationship between health states based on disease activity [11] and utility values in patients with CSU. The objective of the current study was to investigate the relationship between disease activity levels and the associated utility estimates in CSU, as derived from the EQ-5D, and to provide suitable utility value estimates for use in economic models.

\section{Methods}

\subsection{Data}

For the present analyses, patient-level data came from three randomized, double-blind, placebo-controlled, phase 3 clinical trials of omalizumab for the treatment of patients with CSU with inadequate control from the standard of care: ASTERIA I $(N=318)$ [12], ASTERIA II $(N=322)$ [13] and GLACIAL $(N=335)$ [14]. Treatment was administered once every 4 weeks for 24 weeks in the ASTERIA I and GLACIAL trials, and once every 4 weeks for 12 weeks in the ASTERIA II trial. All three trials had a follow-up period of 16 weeks with no active treatment (see Fig. A-1 and Table A-1 in the Electronic Supplementary Material).

\subsection{Measures}

The UAS is a validated daily diary [15], which has two components: the itch severity score and the hives score. Patients filled in the diary twice daily over the entire period of the trials. The UAS7 is a weekly composite score, derived by summing daily average UAS scores over 7 days. The UAS7 is used to determine disease activity and response to treatment [1]. UAS7 scores range from 0 to 42, with higher scores reflecting greater urticaria activity (more severe itch and a larger number of hives). The UAS7 was selected as the primary endpoint for this analysis because it covers the key symptoms and signs of the disease.

UAS7 scores from the three trials were categorized into five urticaria health states: urticaria free (UAS7 $=0)$, wellcontrolled urticaria (UAS7 $=1-6)$, mild urticaria (UAS7 $=7-15)$, moderate urticaria $($ UAS7 $=16-27)$ and severe urticaria (UAS7 $=28-42$ ) [11]. Two of these health states (urticaria free and well-controlled urticaria) were endpoints for the clinical trials. Patients were required to have a UAS7 score of 16 or higher to be included in the trial, and this was considered by medical experts and 
regulatory agencies as the threshold of moderate to severe urticaria, therefore a score of 16 was considered the lower threshold of the moderate urticaria health state. In addition, on the basis of clinical expertise, a score of 28 or higher was considered severe for reimbursement purposes and by the UK National Institute for Health and Care Excellence [16]. Thus, the remaining UAS7 scores (7-15) were used to define the mild urticaria health state. UAS7 scores for every week were available, but scores reported in the 7 days prior to baseline and every study visit were used in the analysis. Study visits were conducted every 4 weeks after baseline until week 40 in the ASTERIA I and GLACIAL trials and until week 28 in the ASTERIA II trial.

The EQ-5D is a generic, standardized instrument used as a measure of health outcomes across many diseases. The measure can describe the effect of different health states on HRQoL. It consists of five questions, each asking about a particular dimension: mobility, self-care, usual activities, pain/discomfort and anxiety/depression [8]. The EQ-5D allows comparison of HRQoL across diseases and facilitates calculation of quality-adjusted life-years (QALYs). The version used in these three clinical trials, the EQ-5D$3 \mathrm{~L}$, has three levels of response for each question, evaluating the respondent's current status: no problems, some problems or extreme problems. A single index value for health states (called a utility) can be calculated from the combination of responses to the five questions [9]. The maximum utility value is 1 (perfect health), and 0 corresponds to death. In some instances, the utility can be less than 0 , indicating a health state that is considered worse than death. As per the trial protocols, in the ASTERIA I and GLACIAL trials, the EQ-5D data were collected at baseline and at weeks 12 and 40; in the ASTERIA II trial, the data were collected at baseline and at weeks 12 and 28 . The EQ-5D index score was constructed using UK population-based weights [9].

\subsection{Analyses}

Descriptive statistics were considered for the baseline characteristics for each trial, including patient age and sex, duration of CSU and other key variables. For descriptive statistics, continuous variables were presented as means, standard deviations (SDs) and ranges; categorical variables were presented as counts and percentages. The mean utilities were calculated for each of the five UAS7 health states by trial and visit. There was no imputation of missing data.

Because the analyses at the individual trial level and individual time points resulted in inconsistent utilities, with variability in the relationship of utilities to disease activity, data from the individual trials and individual time points were pooled to increase the sample size and generate more reliable estimates, and mixed-effects regression models were tested to estimate utilities. A linear mixed-effects model was used to predict EQ-5D utilities for each UAS7 health state on the basis of pooled data from the three trials. The validity of pooling the three trials was considered through visual comparisons and interaction terms to assess differences in the relationship between health states and utilities among the three trials. Random effects for trials and patients were included, along with the following covariates as fixed effects: UAS7 health state at baseline (moderate or severe), presence of angioedema at baseline and during follow-up (a binary variable indicating the presence of 1 or more days with angioedema in the 7 days prior to each of the visits included in the model), duration of CSU, number of previous CSU medications, visit, treatment, and patient age and sex (see Eq. A-1 in the Electronic Supplementary Material). Interaction terms between the duration of CSU and the UAS7 health state and between the presence of angioedema and the UAS7 health state also were considered in the models.

A parsimonious model was selected using an elimination stepwise procedure-a combination of backwards elimination, in which covariates that were not significant were sequentially removed from the model according to their $P$ values $(P<0.05)$, and forward inclusion, in which those variables that had been previously removed were considered for inclusion on the basis of their $P$ values. The UAS7 health state was forced into the model to ensure it was always a predictor. The results of the models with nonsignificant covariates are not shown, as those models were an intermediate step. Consistency was observed in the selection of variables on the basis of their significance across the sensitivity analysis and primary analysis. No significant evidence was found that those variables that were not retained in the final model were predictive of utilities. The model fit was examined by comparing margins over visits, health state, presence of angioedema and trial against the observed values. Margins, or marginal effects, are post-estimation statistics calculated from predictions of a previously fit overall model. Margins were compared with observed group values across visit, health state and trial (because of small subgroups across all covariates). Margins were plotted against a line of best fit.

The primary analysis considered angioedema in the 7 days prior to the EQ-5D utility value. Sensitivity analyses were also conducted, which considered the covariates angioedema at baseline and angioedema between 4 and 12 weeks (the latter was a secondary efficacy endpoint of the studies) with and without the 28-week data from ASTERIA II.

Further sensitivity analyses were conducted to confirm the robustness of the results by evaluating the impact on utilities of including covariates that were not significant in 
one model but were significant in alternative models. This allowed evaluation of the stability of the utility estimates.

In addition, subgroup analysis was conducted to further explore possible differences in the relationship between UAS7 health state and utilities in (1) those patients who had angioedema at baseline and those who did not; (2) those patients who had angioedema at any time during follow-up and those who did not (regardless of their baseline angioedema status); and (3) those patients who were in the severe health state (UAS7 28-42) at baseline and those who were in the moderate health state at baseline (UAS7 16-27).

All analyses were performed using Stata 13 software (StataCorp; College Station, TX, USA).

\section{Results}

The baseline characteristics of patients in the ASTERIA I $(N=318), \quad$ ASTERIA II $(N=322)$ and GLACIAL $(N=335)$ trials were consistent, with similar age distributions, sex distributions and durations of CSU (Table 1). The mean (SD) UAS7 scores ranged from 30.7 (6.8) to 31.1 (6.6), and the mean (SD) EQ-5D score ranged from $0.68(0.27)$ to $0.73(0.24)$.

Calculation of utilities for each UAS7 health state revealed inconsistent utilities for individual treatment arms and at individual time points, with improved response not always equating to an increase in the utility. For example, at 12 weeks in GLACIAL, the utilities for those who were

Table 1 Baseline characteristics of patients in chronic spontaneous urticaria (CSU) clinical trials—all treatment arms pooled

\begin{tabular}{|c|c|c|c|}
\hline Characteristic & ASTERIA I, $N=318$ & ASTERIA II, $N=322$ & GLACIAL, $N=335$ \\
\hline Age [years; mean (SD)] & $41.2(14.5)$ & $42.5(13.7)$ & $43.1(14.1)$ \\
\hline Sex: male $[n(\%)]$ & $87(27.4)$ & $78(24.2)$ & $94(28.1)$ \\
\hline \multicolumn{4}{|l|}{ Weight } \\
\hline Mean $[\mathrm{kg}(\mathrm{SD})]$ & $82.2(21.0)$ & $82.4(21.9)$ & $83.9(22.5)$ \\
\hline Median (kg) & 80.0 & 79.0 & 79.6 \\
\hline Range (kg) & 35 to 138 & 43 to 188 & 46 to 172 \\
\hline$<80 \mathrm{~kg}[n(\%)]$ & $158(49.7)$ & $166(51.6)$ & $168(50.1)$ \\
\hline \multicolumn{4}{|l|}{ Duration of CSU } \\
\hline Mean [years (SD)] & $6.9(9.1)$ & $6.5(8.6)$ & $7.4(9.5)$ \\
\hline Range (years) & 0.5 to 50.5 & 0.5 to 66.4 & 0.5 to 54.1 \\
\hline$>10$ years $(\%)$ & 21.7 & 19.1 & 24.0 \\
\hline \multicolumn{4}{|l|}{ UAS7 } \\
\hline Mean (SD) & $31.1(6.6)$ & $30.7(6.8)$ & $30.9(6.6)$ \\
\hline Range & 16.0 to 42.0 & 16.5 to 42.0 & 16.0 to 42.0 \\
\hline \multicolumn{4}{|l|}{ Weekly itch severity score } \\
\hline Mean (SD) & $14.3(3.5)$ & $14.0(3.7)$ & $14.0(3.6)$ \\
\hline Range & 8.0 to 21.0 & 8.0 to 21.0 & 7.5 to 21.0 \\
\hline Score $<13(\%)$ & 34.0 & 43.8 & 39.1 \\
\hline Presence of angioedema (\%) & 47.5 & 40.7 & 53.1 \\
\hline Previous medications for CSU: overall $[n(\%)]$ & $318(100.0)$ & $322(100.0)$ & $335(100.0)$ \\
\hline Antihistamines & 317 (99.7) & $322(100.0)$ & $335(100.0)$ \\
\hline Histamine $\mathrm{H} 2$ blockers & $93(29.2)$ & $113(35.1)$ & $297(88.7)$ \\
\hline Immunosuppressants & $29(9.1)$ & $26(8.1)$ & $34(10.1)$ \\
\hline Ciclosporin & $26(8.2)$ & $24(7.5)$ & $23(6.9)$ \\
\hline LTRAs & $83(26.1)$ & $83(25.8)$ & $195(58.2)$ \\
\hline Other & $148(46.5)$ & $143(44.4)$ & $197(58.8)$ \\
\hline Dapsone & $5(1.6)$ & $8(2.5)$ & $11(3.3)$ \\
\hline Omalizumab & $4(1.3)$ & $2(0.6)$ & $2(0.6)$ \\
\hline Steroids & $159(50.0)$ & $161(50.0)$ & $194(57.9)$ \\
\hline EQ-5D utility [mean (SD)] & $0.68(0.27)$ & $0.71(0.26)$ & $0.73(0.24)$ \\
\hline Range & -0.08 to 1 & -0.08 to 1 & -0.23 to 1 \\
\hline
\end{tabular}

LTRA leukotriene receptor antagonist, $S D$ standard deviation, UAS7 Urticaria Activity Score over 7 days 
severe at baseline were 0.9 for those with mild urticaria and 0.86 for those with well-controlled urticaria (see Tables A-3, A-4 and A-5 in the Electronic Supplementary Material). This was likely due to the very small numbers of patients in each UAS7 health state.

Pooling of trials to increase the overall sample size, examining utilities across all visits and taking into account angioedema at any point in the trial resulted in more consistent utilities for each health state. The final model (see Table A-2 in the Electronic Supplementary Material) included only the significant covariates of the assessment point (visit) in the trial and angioedema in the 7 days prior to the visit; study and patient were included as random effects. In the pooled analyses of all trials, treatment arms and time points (Fig. 1), patients with greater CSU activity were observed to have lower EQ-5D utilities $(0.710$ for severe urticaria, 0.780 for moderate urticaria, 0.829 for mild urticaria, 0.862 for well-controlled urticaria and 0.894 for urticaria free), and with each improved UAS7 health state, there was an incremental increase in the utility. A comparison of the predicted values with the observed data did not suggest any issues with the model fit and indicated that the model accurately predicted the underlying data (see Figs. A-2 and A-3 in the Electronic Supplementary Material).

All comparisons between EQ-5D utility values, except for well-controlled urticaria versus urticaria free and for well-controlled urticaria versus mild urticaria, showed significant differences between health states at $P<0.005$.

As seen in the primary analysis of all patients and all time points, consistent utilities were observed in the sensitivity analyses (Table 2); patients with more severe CSU had lower EQ-5D utilities; and with each improved UAS7

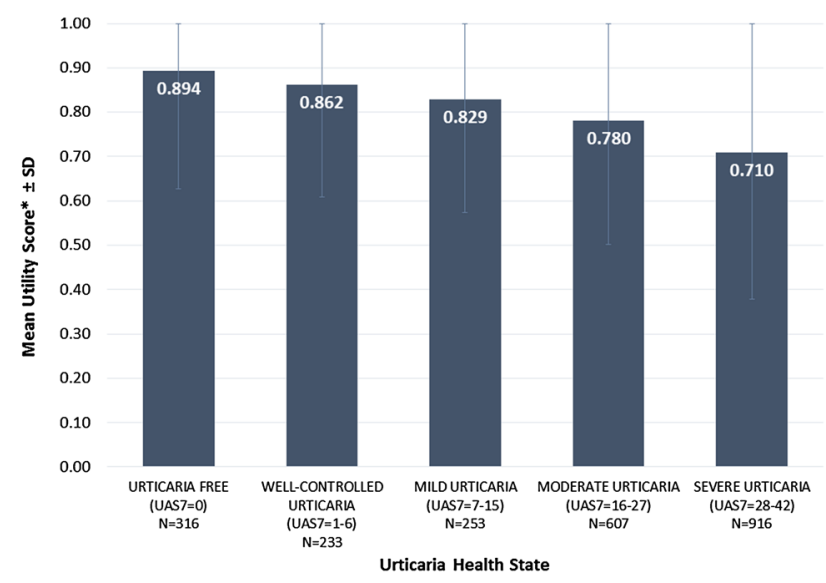

Fig. 1 Utility weights for chronic spontaneous urticaria health states based on Urticaria Activity Score over 7 days (UAS7) pooled data including all patients, all treatment arms and all time points. Note: the upper error bars are capped at 1.00, which represents perfect health; 0.00 represents death. $S D$ standard deviation health state, there was an incremental increase in the utility. Sensitivity analysis 1 , which also included angioedema between weeks 4 and 12 as a covariate and omitted 28-week data from ASTERIA II, provided very similar results, typically differing only at the third decimal place (Table 2). This also was true for sensitivity analysis 2, which considered all time points and also included angioedema between weeks 4 and 12 as a covariate (Table 2).

The results of the subgroup analyses showed a pattern similar to those of the primary and sensitivity analyses, with EQ-5D utilities increasing with decreasing urticaria activity in all subgroups (Table 3). Overall, the presence of angioedema at both baseline and during follow-up decreased utility in all health states, with the exception of angioedema during follow-up in patients with mild and well-controlled urticaria. The utilities of each health state were similar whether the angioedema was present at baseline or during follow-up. While the interaction between the health state and angioedema on utilities was not significant, the largest impact of the presence of angioedema was observed in the severe health state. Similarly, greater CSU activity at baseline decreased the overall utility in the majority of health states.

\section{Discussion and Conclusion}

The results presented here demonstrate that when sample sizes are sufficient (that is, by pooling of trials, treatment arms and time points), consistent EQ-5D utilities for differing levels of disease activity can be obtained. Thus, in contrast to analyses that have focused on treatment arms within a single trial, the pooled trial and treatment arm analyses demonstrated that EQ-5D utilities increase with decreasing urticaria activity. The robustness of the results was confirmed by the sensitivity and subgroup analyses. When the presence of angioedema 7 days prior to a visit was examined at various time points, there were some slight changes in utilities but the general pattern held: the better the CSU health state, as measured by the UAS7, the greater the EQ-5D utility, indicating a greater preference for less active CSU health states. This pattern was also demonstrated in the subgroup analyses. Overall, lower utilities were generally seen in each health state with greater baseline disease activity and in the presence of angioedema.

These analyses document an evaluation of utilities associated with the activity of CSU. The clinical impact of CSU, especially on patients' HRQoL, is immense, demonstrated particularly by lower physical and mental components scores on the SF-36 and higher rates of 
Table 2 Sensitivity analyses with the presence of angioedema at baseline and between 4 and 12 weeks: (1) excluding and (2) including 28-week data from ASTERIA II

\begin{tabular}{llll}
\hline Health state & UAS7 score & EQ-5D score [mean (SD)] & \\
\cline { 3 - 4 } & & $\begin{array}{l}\text { Sensitivity analysis 1: excluding } \\
\text { ASTERIA II 28-week data }\end{array}$ & $\begin{array}{l}\text { Sensitivity analysis 2: including } \\
\text { ASTERIA II 28-week data }^{\mathrm{a}}\end{array}$ \\
\hline Urticaria free & 0 & $0.897(0.255)$ & $0.905(0.253)$ \\
Well controlled & $1-6$ & $0.859(0.244)$ & $0.862(0.243)$ \\
Mild & $7-15$ & $0.845(0.244)$ & $0.840(0.244)$ \\
Moderate & $16-27$ & $0.782(0.261)$ & $0.785(0.262)$ \\
Severe & $28-42$ & $0.712(0.308)$ & $0.712(0.307)$
\end{tabular}

$S D$ standard deviation, UAS7 Urticaria Activity Score over 7 days

a The sensitivity analyses considered the presence of angioedema between 4 and 12 weeks and baseline angioedema as possible covariates in the model. Following backwards elimination, fixed effects for visit and angioedema between 4 and 12 weeks were retained in the model, along with the UAS7 health state. The models also included random effects for study and patient

Table 3 Subgroup analyses of patients with and without angioedema at baseline and during follow-up, and by baseline urticaria activity

\begin{tabular}{|c|c|c|c|c|c|c|c|}
\hline \multirow[t]{4}{*}{ Health state } & \multirow[t]{4}{*}{ UAS7 score } & \multicolumn{6}{|c|}{ EQ-5D score [mean (SD)] } \\
\hline & & \multicolumn{4}{|c|}{ Angioedema during trial } & \multirow{2}{*}{\multicolumn{2}{|c|}{ Baseline urticaria activity }} \\
\hline & & \multicolumn{2}{|l|}{ At baseline } & \multicolumn{2}{|c|}{ During follow-up } & & \\
\hline & & Without & With & Without & With & $\begin{array}{l}\text { Moderate: UAS7 } \\
\text { score 16-27 }\end{array}$ & $\begin{array}{l}\text { Severe: UAS7 } \\
\text { score } 28-42\end{array}$ \\
\hline Urticaria free & 0 & $0.904(0.225)$ & $0.902(0.276)$ & $0.908(0.229)$ & $0.896(0.280)$ & $0.913(0.241)$ & $0.903(0.246)$ \\
\hline Well controlled & $1-6$ & $0.869(0.224)$ & $0.857(0.254)$ & $0.856(0.224)$ & $0.865(0.264)$ & $0.876(0.235)$ & $0.871(0.235)$ \\
\hline Mild & $7-15$ & $0.838(0.223)$ & $0.832(0.261)$ & $0.831(0.222)$ & $0.836(0.269)$ & $0.830(0.236)$ & $0.853(0.237)$ \\
\hline Moderate & $16-27$ & $0.795(0.224)$ & $0.760(0.285)$ & $0.790(0.221)$ & $0.774(0.310)$ & $0.759(0.346)$ & $0.800(0.241)$ \\
\hline Severe & $28-42$ & $0.735(0.253)$ & $0.673(0.349)$ & $0.755(0.255)$ & $0.679(0.374)$ & $0.767(0.195)$ & $0.700(0.286)$ \\
\hline
\end{tabular}

$S D$ standard deviation, UAS7 Urticaria Activity Score over 7 days

depression, anxiety and sleep difficulties in patients with CSU than in those without the disease [4]. In addition, evaluation of EQ-5D utility scores is of great importance, as it allows comparison of HRQoL across diseases and enables calculation of QALYs, which are used in economic models. QALYs are essential components of health technology assessments, which support decision making in health care resource allocation.

As with any study, there were potential limitations to this work. First, the version of the EQ-5D used in these three clinical trials was the EQ-5D-3L, and there may be some question about whether it is sensitive enough to capture differences between patients' preferences for one health state versus another. The five CSU health states showed consistent differences in utilities, and so for each successively improved CSU health state, the EQ-5D utility increased from one health state to another-by between 3 and $9 \%$ in the final model. However, sensitivity often is a criticism of the EQ-5D-3L. While there was a clear relationship between the CSU health state and EQ-5D utility in the present case, it is possible that the EQ-5D-3L may not be sufficiently sensitive to capture the full impact of CSU-for example, for patients in the severe UAS7 health state, the utility was 0.710 , which may be higher than would be expected for this subgroup. The recently developed EQ-5D-5L [10] provides measurement properties superior to those of the EQ-5D-3L. The second limitation was that the EQ-5D scores were calculated on observed data. The results may not completely reflect the health utilities of all trial patients, particularly those with more severe disease activity.

A third potential question can be raised about the validity of the CSU health states themselves. However, other work $[7,11]$ has shown that the five health states, as defined here in our models, can discriminate significantly between patients in terms of their Dermatology Life Quality Index scores, sleep interference and interference with daily activities. Thus, any concerns about the validity of these health states should be minimal. Further validation work is ongoing. 
It is essential that health economic researchers and health technology assessment bodies have the latest information in order to determine the cost effectiveness of treatments. In the present case, we now have important information that can assist in determination of the cost effectiveness of CSU treatments and can aid decision makers in their consideration of the reimbursement for such treatments.

The results of these analyses document the utility associated with the severity of CSU health states and suggest that the EQ-5D utility score increases with decreased disease activity. Overall, lower utilities were generally seen in each health state with greater baseline disease activity and in the presence of angioedema.

Acknowledgments All co-authors had involvement in all aspects of the manuscript, with one exception: Donald Stull and Emma Hawe share responsibility for the data analysis. However, the results were shared with all co-authors at each step, and their input was sought regarding the discussion and implications of the results. All authors approved the final version of the manuscript prior to its submission.

\section{Compliance with Ethical Standards}

This study was funded by Novartis Pharma AG.

Conflict of interest Emma Hawe, Doreen McBride and Donald Stull are employees of RTI Health Solutions, which provides consulting and other research services to pharmaceutical, device, government and nongovernment organizations. In their salaried positions, they work with a variety of companies and organizations. They receive no payment or honoraria directly from these organizations for services rendered. Maria-Magdalena Balp is an employee of Novartis Pharma AG. Haijun Tian is an employee of Novartis Pharmaceuticals Corporation. Anna Halliday is an employee of Novartis Pharmaceuticals UK Limited.

Open Access This article is distributed under the terms of the Creative Commons Attribution-NonCommercial 4.0 International License (http://creativecommons.org/licenses/by-nc/4.0/), which permits any noncommercial use, distribution, and reproduction in any medium, provided you give appropriate credit to the original author(s) and the source, provide a link to the Creative Commons license, and indicate if changes were made.

\section{References}

1. Zuberbier T, Aberer W, Asero R, et al. The EAACI/GA2LEN/ EDF/WAO guideline for the definition, classification, diagnosis, and management of urticaria: the 2013 revision and update. Allergy. 2014;69(7):868-87.

2. Maurer M, Magerl M, Metz M, et al. Revisions to the international guidelines on the diagnosis and therapy of chronic urticaria. J Dtsch Dermatol Ges. 2013;11(10):971-8. doi:10.1111/ ddg. 12194.
3. Maurer M, Weller K, Bindslev-Jensen C, et al. Unmet clinical needs in chronic spontaneous urticaria: a GA2LEN Task Force report. Allergy. 2011;66:317-30.

4. Balp MM, Vietri J, Tian H, Isherwood G. The impact of chronic urticaria from the patient's perspective: a survey in five European countries. Patient. 2015;8(6):551-8.

5. O'Donnell BF, Lawlor F, Simpson J, et al. The impact of chronic urticaria on the quality of life. $\mathrm{Br} \mathrm{J}$ Dermatol. 1997;136(2):197-201.

6. Staubach P, Eckhardt-Henn A, Dechene M, et al. Quality of life in patients with chronic urticaria is differentially impaired and determined by psychiatric comorbidity. $\mathrm{Br} \mathrm{J}$ Dermatol. 2006;154(2):294-8.

7. Stull DE, McBride D, Gimenez-Arnau A, et al. Validation of chronic spontaneous/idiopathic urticaria (CSU/CIU) health states using weekly Urticaria Activity Score (UAS7) and Dermatology Life Quality Index (DLQI) [poster]. American Academy of Dermatology Annual Meeting, San Francisco, March 2015. RTI Health Solutions. 2015. https://www.rtihs.org/publications/ validation-chronic-spontaneousidiopathic-urticaria-csuciu-healthstates-using-weekly. Accessed 10 Oct 2015.

8. Gusi N, Olivares PR, Rajendram R. The EQ-5D health-related quality of life questionnaire. In: Preedy VR, Watson RR, editors. Handbook of disease burdens and quality of life measures. New York: Springer; 2010. p. 87-99.

9. Kind P, Dolan P, Gudex C, Williams A. Variations in population health status: results from a United Kingdom national questionnaire survey. BMJ. 1998;316:736-41.

10. EuroQol Research Foundation. EuroQoL EQ-5D-5L. EuroQol Research Foundation. 2015. http://www.euroqol.org/faqs/eq-5d5l.html. Accessed 1 Oct 2015.

11. Stull DE, McBride D, Gimenez-Arnau A, et al. Categorical health states in chronic spontaneous urticaria (CSU) based on the weekly Urticaria Activity Score (UAS7): are they distinct, discriminative, and reproducible? [poster]. ISPOR 17th Annual European Congress, Amsterdam, November 2014. RTI Health Solutions. 2014. https://www.rtihs.org/sites/default/files/ISPOR\% 202014_CSU\%20Health\%20states\%20Final\%20version\%20for\% 20print_862_6th\%20Nov.pdf. Accessed 6 Oct 2015.

12. Saini S, Rosen $\mathrm{K}$, Hsieh $\mathrm{H}$, et al. Efficacy and safety of omalizumab in patients with chronic idiopathic urticaria who remain symptomatic despite concomitant $\mathrm{H} 1$ antihistamine therapy: results of a phase 2 trial [abstract]. 30th Congress of the European Academy of Allergy and Clinical Immunology, Istanbul, June 2011. Allergy. 2011;66:33.

13. Maurer M, Rosen K, Hsieh HJ, et al. Omalizumab for the treatment of chronic idiopathic or spontaneous urticaria. N Engl J Med. 2013;368:924-35.

14. Kaplan A, Ledford D, Ashby M, et al. Omalizumab in patients with symptomatic chronic idiopathic/spontaneous urticaria despite standard combination therapy. J Allergy Clin Immunol. 2013;132:101-9.

15. Mathias SD, Dreskin SC, Kaplan A, et al. Development of a daily diary for patients with chronic idiopathic urticaria. Ann Allergy Asthma Immunol. 2010;105(2):142-8.

16. National Institute for Health and Care Excellence. Omalizumab for previously treated chronic spontaneous urticaria. NICE technology appraisal guidance [TA339]. National Institute for Health and Care Excellence. 2015. https://www.nice.org.uk/guidance/ ta339/chapter/1-guidance. Accessed 6 Oct 2015. 